\title{
Gold Alloys with Shape-memory
}

\author{
G. B. Brook
}

Fulmer Research Institute, Stoke Poges, England

\begin{abstract}
Among a relatively few metallic systems certain alloys of gold exhibit a remarkable mechanical memory. After severe distortion a change of temperature will cause them to return to their original shape. This phenomenon is reviewed, and some possible applications are outlined for gold alloys having this unusual property in addition to their excellent resistance to corrosion.
\end{abstract}

Twenty years have now passed since the experiments were carried out at Columbia University by Professor T. A. Read and Dr L. C. Chang which demonstrated that a reversible shape change, often known as shape-memory, could be obtained in single crystals of gold-cadmium alloys. Shape-memory alloys can be deformed at one temperature to form a new shape and can be restored to their original shape spontaneously by heating to above a critical temperature. This temperature can be varied by altering the composition of the alloy so that material can be produced which will change shape at any particular operating temperature within certain limitations dictated by the nature of the alloy system.

Single crystals are expensive to produce and, in the case of gold-cadmium alloys, are brittle as well. Thus shape-memory has remained a scientific curiosity for many years. But what if shape-memory could be produced in gold alloys with the ductility and malleability usually taken for granted in these materials? What possibilities does this open up for using gold? First let us consider what shape-memory alloys are.

Alloys which exhibit the shape-memory effect undergo a low energy, self-accommodating transformation to a martensitic phase on cooling through a critical temperature known as the $M s$ temperature. In the absence of stress, this is associated with a small, over'-all shape change.

The reversible martensitic transformation can be observed readily by measuring changes in resistivity as the temperature is changed through the critical temperature range. As shown in Figure 1, on cooling a gold alloy containing copper and zinc a sharp rise in resistance occurs at the $M s$ temperature. On completion of the transformation at the Mf temperature, the resistance again falls as the martensite is cooled. On heating the martensitic phase, reversal to the high temperature form starts at the As temperature and is completed at the Af temperature.
The reason why the shape change is small when the alloy is cooled through the critical Ms temperature can be best explained by considering the microstructure of an alloy with a self-accommodating martensitic transformation such as 50 atomic per cent gold-manganese, shown in Figure 2. As the alloy transforms to martensite, the structure shears first

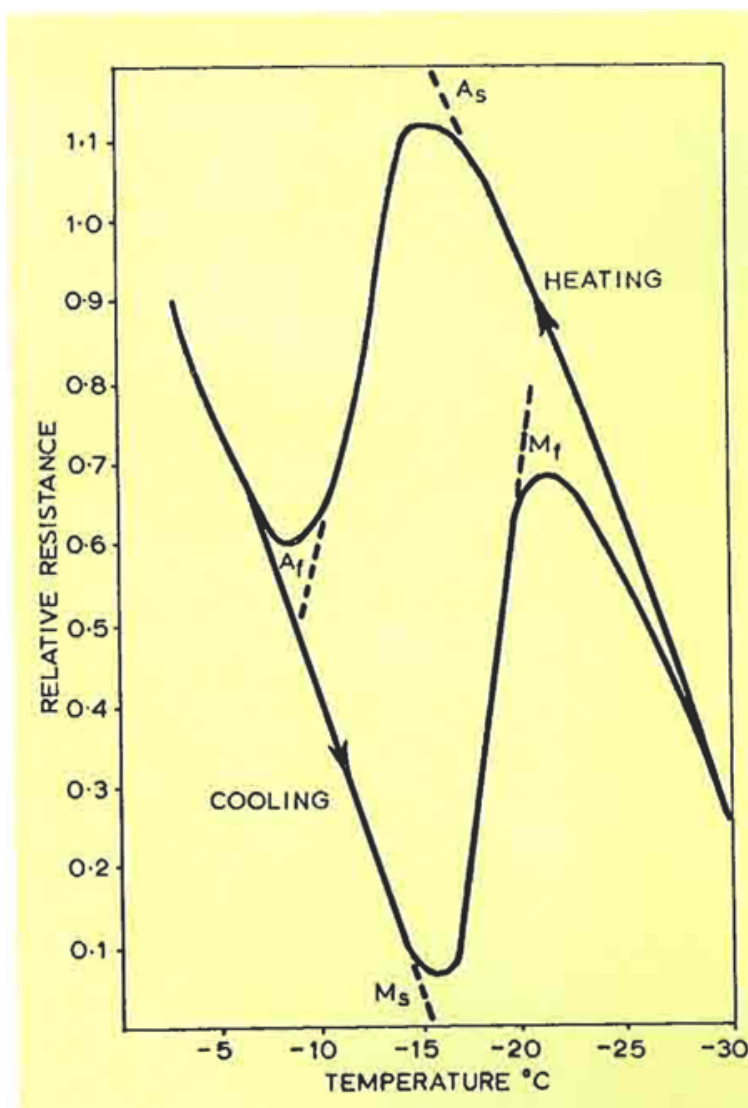

Fig. 1 Transformation characteristics of a goldcopper-zinc alloy. The reversible martensitic transformation for the shape-memory effect can be readily observed by measuring changes in resistivity with temperature 
Fig. 2 The microstructure of 50 atomic per cent goldmanganese, showing the banled martensitic structure obtained by quenching to 20 leg C below its Ms temperature. This type of structure is ideally suited for the development of the shape-memory effect $\times \mathbf{1 6 0}$ Photomicrograph kindly supplicd by Professor P. Gaunt of the University of Manitoba

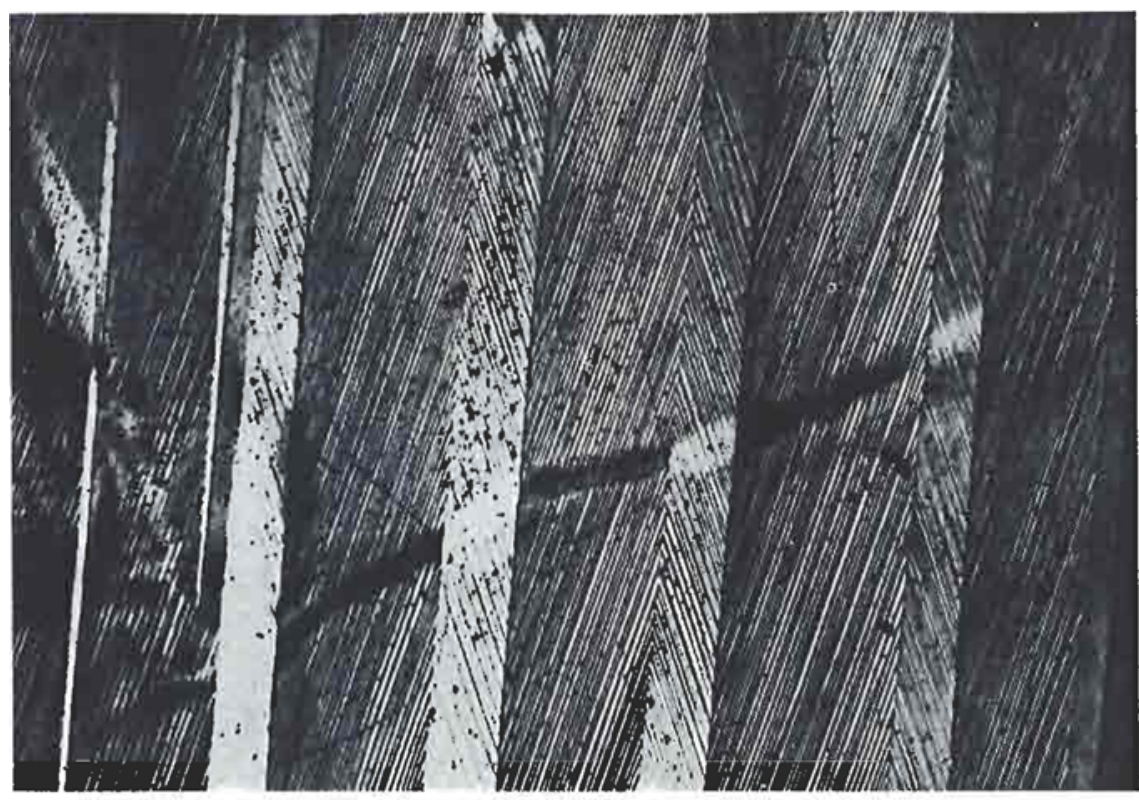

one way and then the other so that the deformation in alternative bands almost cancels out. The behaviour is like that of a pack of playing cards; if one slides successive cards in a pack of playing cards $\frac{1}{8}$ in. in alternative directions the overall shape change is small. If the gold alloy of Figure 2 is transformed under stress or if the martensite is deformed, it is found that the boundaries between the parallel bands are mobile and it is possible to make the bands causing shear in one direction to disappear and those giving shear in the opposite direction to double in thickness, so that instead of the deformation from one band cancelling that of its neighbour, they all now reinforce each other and make a considerable deformation. In our playing card analogy, each card is now made to slide ${ }_{i}^{1}$ in in the same direction as its neighbour so that each pair of cards contributes $\frac{1}{2}$ in instead of zero. If one considers the whole pack of cards, a movement of $6 \frac{1}{2}$ inches is possible.

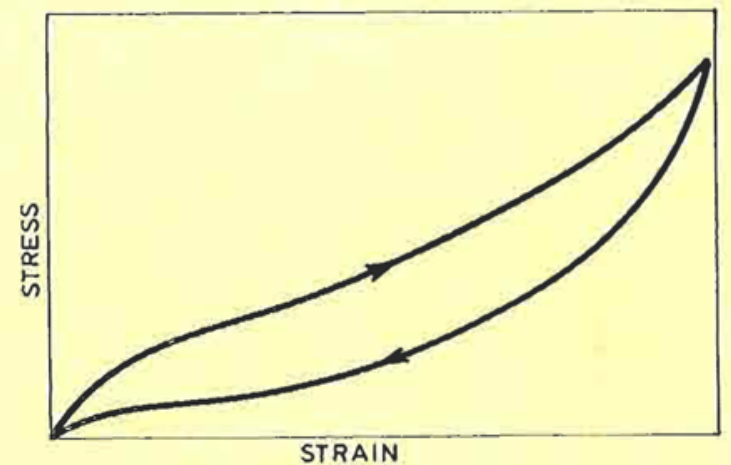

(a) Temperature above $\mathrm{Ms}_{\mathrm{s}}$
If the deformed martensitic structure is reheated through its As-Af temperature range, the martensite transforms to the original high temperature phase and so the shape change imparted to the martensite is also reversed just as one can snap the pack of cards back to its original shape.

If the alloy is deformed just above the Ms temperature while it is still in the high temperature form, it first extends elastically in Hookean mode but at a relatively low stress, the martensitic transformation can be induced and the alloy extends rapidly with litte increase in load, as shown in Figure 3a. Some alloys can be extended to 15 to 20 per cent in this manner and on unloading revert to their original length as the martensite transforms back to the original structure.

This unusual elastic behaviour above the Ms temperature has been called "super-elasticity" and is possible in all shape-memory alloys.

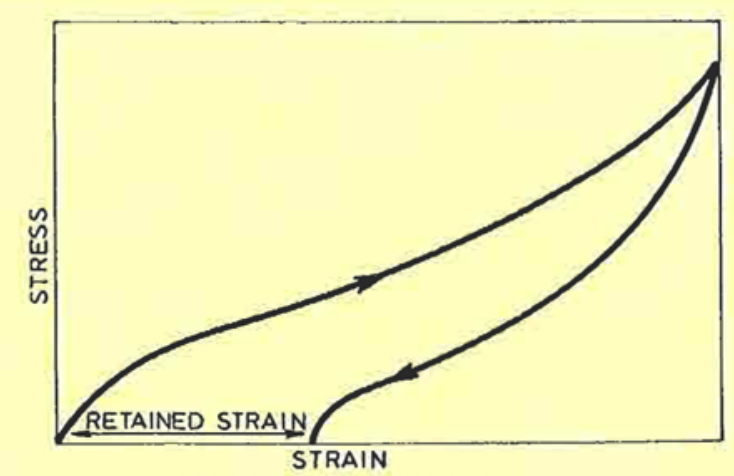

(b) Temperature below $\mathrm{Ms}_{\mathrm{s}}$

Fig. 3 Stress-strain curves for ferro-elastic materials deformed (a) above and (b) below the Ms temperature. On heating to above the Af temperature the retained strain in (b) is released and the original dimensions of the speeimen are restored 


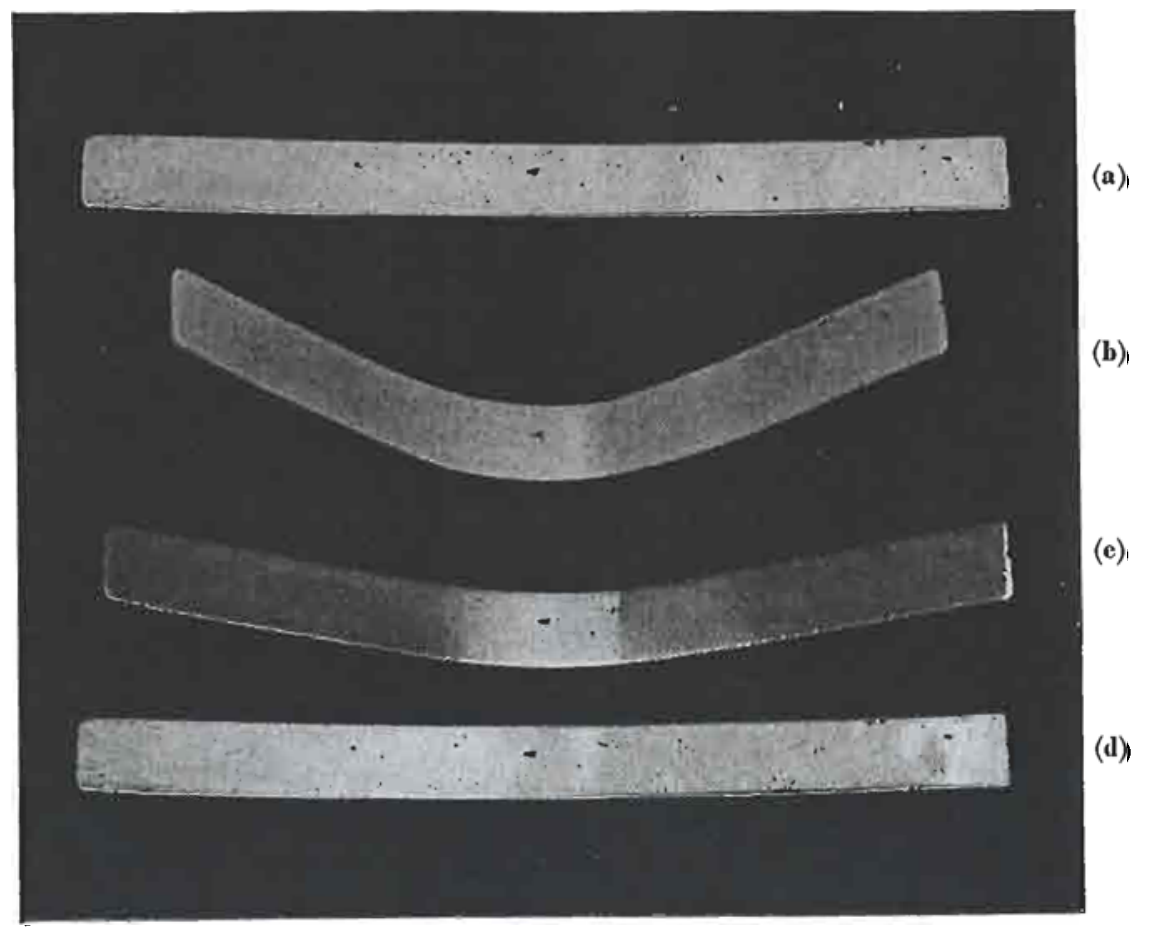

Fig. 4 The shape-memory effect produced by deforming rolled strips of a gold-copperzinc alloy. At room temperature the spccimen (4a) was straight and remained straight on cooling to $-30^{\circ} \mathrm{C}$, below its martengitic temperature. The specimen was hent to approximately $60^{\circ}$ at $-30^{\circ} \mathrm{C}$ (4b) and then allowed to warm back towards room temperature. At $-12^{\circ} \mathrm{C}$, between the As and Af temperatures, the angle of bend was reduced to $30^{\circ}(4 \mathrm{c})$, and at $20^{\circ} \mathrm{C}$, above the Af temperature, it had recovered its original shape (4,l)

When the alloy is deformed below the Ms temperature, a similar stress-strain curve is obtained (Figure 3b) as the martensite structure is adjusted, but on unloading only part of the strain is recovered elastically and the remainder is retained as plastic strain in the deformed martensite. On heating to above the Af temperature, this retained strain is released and the original dimensions of the specimen restored as the structure reverts to its original form. In a ductile alloy, as much as 12 per cent strain can be reversed in this way. It is this strain, or the stress which develops if the alloys are prevented from recovering, which can be made use of in practical devices.

The behaviour of these alloys in compression mirrors that in tension and so closed hysteresis loops can be obtained in stress-strain curves in which high hysteresis losses occur below the Mf temperature and low losses above the Ms. The analogy with hysteresis curves in ferro-magnetic and ferro-electric materials has led to the use of the term "ferro-elasticity" to describe the behaviour of shape-memory alloys. The existence of this hysteresis loop also means that the alloys have high damping capacity which provides another use for them.

Ferro-elastic behaviour is most frequently found in intermetallic compounds like AuCd but, like AuCd, they are usually too brittle for commercial use, even though patents have been granted for devices made in this alloy. It was not until shape-memory was discovered in the compound $\mathrm{TiNi}$, which is more ductile even in polycrystalline form, that industrial use of memory alloys became possible. Devices relying on the shape memory of TiNi are now marketed by the Raychem Corporation of California. Now research at Fulmer Research Institute in recent years has led to the discovery of a range of ductile and malleable alloys exhibiting shape-memory for which patents have either been granted or applications are pending in many countries. It has been possible to predict that several gold alloys should exhibit shapememory without displaying the extreme brittleness of gold-cadmium single crystals.

Not only do these alloys undergo the shapememory change in polycrystalline forms as well as single crystals, they can also be melted and cast and then fabricated to rod, sheet or wire by conventional methods. Although most of the ferro-elastic materials are intermetallic compounds which are inherently brittle, it is possible to develop alloys which are ductile in the form in which they will be used as components.

An example of a gold-copper-zinc alloy that undergoes shape-memory is shown in Figure 4. In the top photograph the strip is in its high temperature straight form. After cooling to $-60^{\circ} \mathrm{C}$ to below the $\mathrm{Mf}$ temperature, it is bent to an angle of about $60^{\circ}$ (upper middle photograph). On allowing to warm up, the strip begins to straighten at $-12^{\circ} \mathrm{C}$ which is above the As temperature (lower middle photograph) and recovers its original shape at $20^{\circ} \mathrm{C}$ above the Af temperature. (The composition of this alloy has to be controlled otherwise brittle intermetallic phases can be formed; it is the presence of such a phase which is responsible for the small crack visible in Figure 4.) 
WIRE

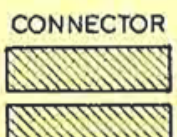

Fig. 5 One possible application of shape-memory in gold alloys lies in the field of connectors. In this example the internal diameter of the connector is initially less than that of the wire

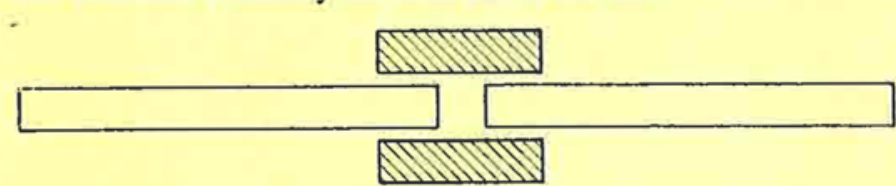

By expanding the connector at low temperature the wires can be inserted

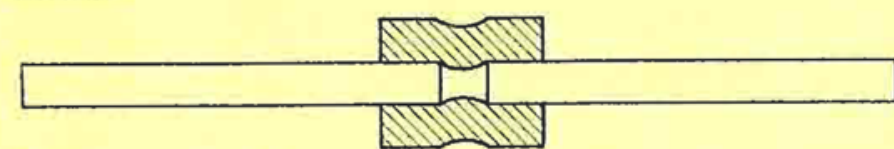

During warming back to room temperature the connector contracts on to the wires. Similar applications of the shape memory effect eould be achieved with strips and other shapes new type of wire connector such as that illustrated in the three stages of Figure 5

Here it can be seen that by preventing the alloy from shrinking completely to its original size, the connector can be used to clamp the wire with considerable force. Other possible devices can be imagined.

Gold memory alloys can be used to expand further its use in jewellery manufacture for gripping jewels in mounts. Memory alloys can be used for thermal switches. They can be used to perform movements in inaccessible places, for example, as body implants where a body heat can be used to release the movement. Uses can also be found in dentistry.

Why should one use gold memory alloy? Gold alloys will be preferred for those applications where the colour, and the corrosion- and tarnish-resistance of gold is important. The electrical conductivity of gold memory alloys is about 25 per cent IACS. Thus gold memory alloys could well be used to make a
The realisation of what these new and unusual memory materials can do will create new ideas in the minds of designers; the addition of the property of shape-memory to the unique properties of gold alloys will ensure that there will be further uses for gold in industry.

\section{Structural Changes in Gold-Palladium Alloys}

Metallurgy is becoming increasingly a study of imperfections, and there can be few supposedly solid solution systems in which minor departures from the ideal state have not yet been detected. Although studies of this type are usually only of theoretical significance, they assume considerable importance when the alloys being investigated are those in the gold-palladium system.

Gold-palladium alloys are capable of generating high thermo-electric forces and are therefore extensively employed in temperature measurement. The high electrical stability exhibited by these solid solutions appears to indicate complete freedom from gross metallurgical transformations, although evidence for the existence of low temperature ordering reactions has been advanced. Such reactions influence very slightly the thermo-electric properties of the alloy.

Very sensitive experimental methods have hitherto been required to detect the subtle changes that occur, and because of the very sluggish nature of these transformations, electrical resistivities have been carefully monitored over prolonged periods. Recent work carried out by P. V. Valembois at the University of Pennsylvania and reported in his Ph.D. thesis has shown, however, that by cooling fine wires at a rate of $10^{5} \mathrm{deg} \mathrm{C} /$ second large vacancy concentrations can be quenched into these alloys. The tremendous low temperature mobility thus conferred allows short-range ordering processes to proceed more rapidly, and thus facilitates the attainment of true equilibrium.
Alloys containing 2, 5, 10, 20 and 50 atomic per cent of palladium were examined. All those containing 5 per cent or more of palladium exhibited some isothermal resistivity increments which suggested the progress of ordering reactions. A complete correlation between alloy composition and the tendency to order could not unfortunately be established, largely because in the higher palladium alloys the effects caused by vacancy annihilation tended to oppose those caused by ordering. The 20 and 50 atomic per cent palladium alloys did appear, however, to show larger increases in resistance on isothermal annealing than the more dilute alloys.

Although the results of this investigation do not indicate those ranges of alloy compositions from which high stability thermocouple limbs might advantageously be selected, they do allow some very general conclusions to be arrived at. Thus a slight short range ordering tendency appears to exist over the complete gold-palladium binary system, although this reaction can be completely suppressed by quenching. Ordering during subsequent heat treatment is considerably accelerated by the presence of quenched-in vacancies, and this leads to an idea which could be of direct practical value to those concerned in the manufacture of precision resistances. These are frequently aged for stabilisation purposes after manufacture, and $\mathrm{Dr}$ Valembois suggests that the effectiveness of this procedure might be considerably improved by winding the resistor from previously quenched wire.

A.S.D. 